\title{
PHOTOELECTRIC CHARGING OF DUST GRAINS IN THE ENVIRONMENT OF YOUNG STELLAR OBJECTS
}

\author{
Andreas Pedersen ${ }^{1,2,3}$ And Ana I. Gómez de Castro ${ }^{1,4}$ \\ ${ }^{1}$ Instituto de Matemática Interdisciplinar, Universidad Complutense de Madrid, Spain \\ ${ }^{2}$ School of Science and Engineering, Reykjavik University, 101 Reykjavík, Iceland \\ ${ }^{3}$ Science Institute, University of Iceland, 107 Reykjavík, Iceland \\ ${ }^{4}$ S. D. Astronomía y Geodesia Fac. de CC Matemáticas, Universidad Complutense, 28040 Madrid, Spain \\ Received 2010 August 18; accepted 2011 July 15; published 2011 October 3
}

\begin{abstract}
The evolution of disks around young stellar objects (YSOs) is deeply affected by the YSOs' ultraviolet (UV) radiation field especially in the 500-1100 $\AA$ spectral range. The two dominant processes are: the photodissociation of $\mathrm{H}_{2}$ molecules in the Werner and Lyman bands, and the emission of photoelectrons from dust grains when high energy photons are absorbed. Photoelectrons are an important source of gas heating. In this paper, dust grain charging when exposed to various possible UV fields in the YSOs' environment is investigated. Numerical simulations of the evolution of photoelectrons in the electric field created by the charged dust grains are carried out to obtain the charging profile of dust grains. From the simulations it appears that the different spectra produce significant quantitative and qualitative differences in the charging processes. Both the UV background and the AeHerbig star radiation field produce a relatively slow charging of dust grains due to the low fraction of sufficiently energetic photons. The radiation field of T Tauri stars (TTSs) is harder due to the release of magnetic energy in the dense magnetospheric environment. These numerical results have been used to propose a new simple analytical model for grain charging in the atmosphere of protostellar disks around TTSs susceptible to be used in any disk modeling. It has been found that the yield decreases exponentially with the dust charge and that two populations of photoelectrons are produced: a low energy population with mean kinetic energy $E=2.5 \mathrm{eV}$ and a high energy population with $E=5.5-6 \mathrm{eV}$; the energy dispersion within the populations is $\sim 1.3 \mathrm{eV}\left(T \sim 1.5 \times 10^{4} \mathrm{~K}\right)$. The high energy population is susceptible of dissociating the $\mathrm{H}_{2}$ and ionizing some low ionization potential species, such as the Mg. These results add an additional role to dust on the chemistry of the layers just below the $\mathrm{H}_{2}$ photoionization front. This photoelectic yield has been applied to a simple evaluation of the dust charge in the atmospheres of accretion disks ( $\alpha$-disks).
\end{abstract}

Key words: dust, extinction - ISM: general - stars: variables: T Tauri, Herbig Ae/Be - ultraviolet: ISM

\section{INTRODUCTION}

UV radiation strongly affects matter, and the circumstellar environments around young stellar objects (YSOs) are very sensitive to it. Of special interest is the interaction between the stellar ultraviolet (UV) radiation field and the accretion disk that channels mass infall onto the star.

In the diffuse atmosphere of the disk, the dominant interaction is the absorption of UV photons by the $\mathrm{H}_{2}$ molecule in the Lyman and Werner bands; about $15 \%$ of the photons cause photodissociation of the rather inert $\mathrm{H}_{2}$ molecule (Draine \& Bertoldi 1996), leading to both the formation of a photoionization front that propagates inward the disk and an outward photoevaporative flow on the surface, which carves the disk and produces a slow thermal wind (Hollenbach \& Gorti 2009), which has a fundamental role in disk evolution (Alexander et al. 2006). Indirect evidences of the absorption of UV radiation in young planetary disks come from the detection of $\mathrm{H}_{2}$ electronic transitions that are pumped by the Lyman $\alpha$ radiation from the star (Herczeg et al. 2002).

Though $\mathrm{H}_{2}$ by far is the main component of molecular clouds and protostellar disks - the gas to dust mass ratio is $\sim 100$ and $\mathrm{CO} / \mathrm{H}_{2} \sim 7 \times 10^{-5}$-dust plays a relevant role as a source of extinction and free electrons, not to mention its role in disk chemistry. The largest cross section of a dust grain arises when interacting with radiation at wavelengths $\sim 750 \AA$ (declining both toward X-rays and optical wavelengths); thus, dust is more opaque to the optical/ultraviolet stellar spectrum than to the infrared radiation produced by itself. Furthermore, the interaction between dust grains and the UV radiation field result in the generation of a population of high energy photoelectrons. There is evidence of such hot electrons coexisting with molecular hydrogen around T Tauri stars (TTSs; Ingleby et al. 2009) and in the shocks of YSOs' outflows with the environment, like in the Herbig-Haro object HH 2. As an example, the observed $\mathrm{H}_{2}$ emission spectrum of $\mathrm{HH} 2 \mathrm{H}$ can be fitted by radiation from $\mathrm{H}_{2}$ at $1000 \mathrm{~K}$ that is collisionally excited by $20 \mathrm{eV}$ electrons (Raymond et al. 1997). There are, however, two problems with this interpretation. First, it is unclear how hydrogen molecules can mix with hot electrons and radiate without dissociating. Second, the $\mathrm{H}_{2}$ temperature must be kept above $\sim 1000 \mathrm{~K}$ to excite the vibrational levels and below $\sim 10,000 \mathrm{~K}$ to avoid dissociation. Photoelectrons from UV irradiated dust could provide the needed thermostat, given that they are sufficiently energetic. Heating of the gas by collisions with photoelectrons depends on their emission rate, $J_{e}$, and their kinetic energy through:

$$
\Gamma=4 n_{H} \sigma \int_{E_{\min }}^{E_{\max }}(E-e U) J_{e} d E
$$

where $(E-e U)$ is the kinetic energy of an escaping photoelectron, $E$ is the energy of the impinging photons and $U$ the barrier to escape the grain potential, and $n_{H}$ and $\sigma$ are the number density of $\mathrm{H}_{2}$ and the collision cross section of $\mathrm{H}_{2}$ molecules with the photoelectrons, in the simplest version of a dust grains fluid embedded in molecular gas. It should be noted that the kinetic energy of the photoelectrons depends on their interaction with 
the electric field generated by the charged electric grains and it is directly related to the grain charging profile.

In this paper, the results from detailed numerical simulations of the charging process of dust grains are reported for various UV radiation fields of interest for star formation research (photoelectric effects by X-rays and Auger effects are beyond the scope of this paper). Also, a simple parameterization of $J_{e}$, the photoelectric current produced by a dust grain, is derived for grains submitted to the irradiation of the magnetosphere of a TTS. The dependence of the photoelectric yield on the dust grain charge is analyzed in Section 4, and the population of ejected photoelectrons is characterized in terms of its kinetic energy. Finally, the relevance of the photoelectric charging of dust in the atmospheres of $\alpha$-disks is discussed.

\section{NUMERICAL SIMULATIONS}

The applied scheme to account for the interaction between incoming photons and dust grains follows four steps.

1. Capture of ionizing photons by the dust grain.

2. Yield of photoelectrons released from the surface of the grain-core.

3. Determination of the initial kinetic energy of a released photoelectron.

4. Dynamics of the electrons in the local electric field that results from the charged grain-core and the surrounding electronic cloud.

The first three phases are based on the scheme originally proposed by Draine (1978) for the treatment of the interaction between radiation and dust grains. All interactions are parameterized for the most common silicate dust grains (see Pollack et al. 1994; Sargent et al. 2009).

\subsection{Photon Capture}

For a photon with wavelength $\lambda$, the cross section for capture/ absorption is given by

$$
\sigma\left(r_{\text {grain }} / \lambda, \Im(\tilde{n})\right)=Q_{\text {abs }}\left(r_{\text {grain }} / \lambda, \Im(\tilde{n})\right) \pi r_{\text {grain }}^{2},
$$

where $\tilde{n}$ is the complex refractive index for the grain material and $r_{\text {grain }} / \lambda$ is the ratio between the grain size and the wavelength of the impinging photon. The absorption efficiency $Q_{\text {abs }}$ is obtained by applying the Mie theory using the functional form of the refraction index for silicate as stated by Draine (2003).

\subsection{Yield of Photoelectrons}

For a bulk sample, the electron yield, $Y$, produced by a photon is approximated following the scheme by Weingartner \& Draine (2001):

$$
Y(\Theta)=\frac{\alpha\left(\Theta / \chi_{\mathrm{mol}}\right)}{1+\beta\left(\Theta / \chi_{\mathrm{mol}}\right)},
$$

where $\Theta=h v-\chi_{\mathrm{mol}}$, and the parameters $\chi_{\mathrm{mol}}=8 \mathrm{eV}, \alpha=0.5$, and $\beta=5$ represent silicates. For this yield model to be valid, the impinging photon must interact with a molecular orbital and its energy must be $<\chi_{\text {atom }}$. For the more energetic photons, a decrease in yield occurs since they interact with the atomic orbitals, which results in a weaker coupling. To account for this behavior we suggest a simple and analytic approximation:

$$
Y(\Upsilon)=\left(\frac{\Upsilon-a}{a}\right)^{b}
$$

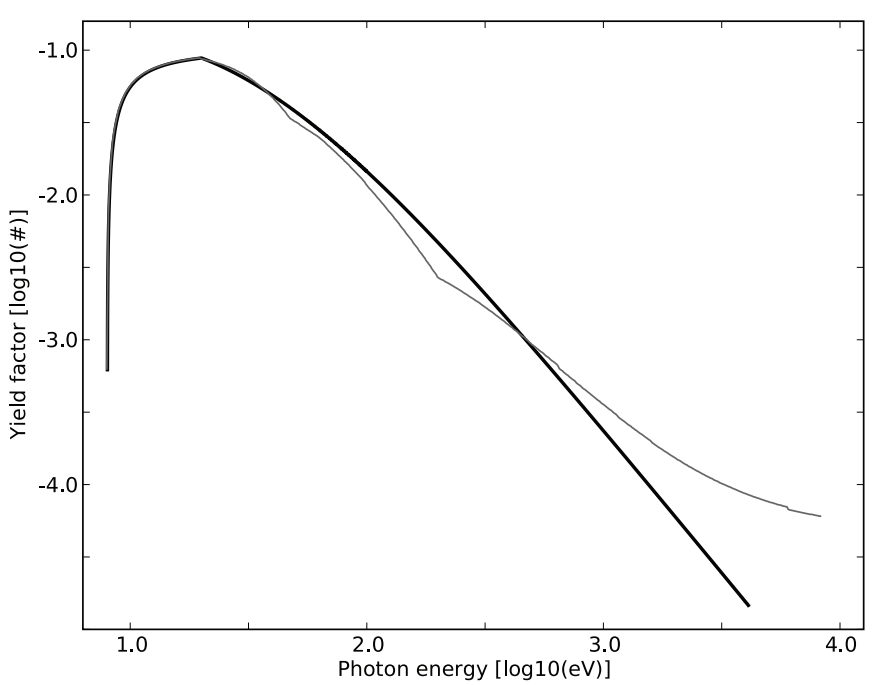

Figure 1. Yield as a function of photon energy. The gray curve is a model by Weingartner et al. (2006) for the decrease at high photon energies, and the bold black curve is the new proposed approximate model, Equation (4).

where $\Upsilon=h v-\chi_{\text {atom }}$ and $a$ and $b$ are fitting parameters. The values used for silicates are $\chi_{\text {atom }}=a=20 \mathrm{eV}$ and $b=-2$. Using these values, a good fit to the more accurate model by Weingartner et al. (2006) is obtained; see Figure 1. With this additional term the full expression for the yield of photoelectrons from a bulk sample becomes

$$
Y(h v)= \begin{cases}\frac{\alpha\left(\Theta / \chi_{\mathrm{mol}}\right)}{1+\beta\left(\Theta / \chi_{\mathrm{mol}}\right)} & \text { if } \chi_{\mathrm{mol}}<\mathrm{h} v \leqslant \chi_{\text {atom }} \\ \frac{\alpha\left(\Theta / \chi_{\mathrm{mol}}\right)}{1+\beta\left(\Theta / \chi_{\mathrm{mol}}\right)}\left(\frac{\gamma-a}{a}\right)^{b} & \text { if } \chi_{\text {atom }}<\mathrm{h} \nu\end{cases}
$$

Size effects result in a dependency between yield and the curvature of the grain surface as an increased curvature results in a stronger electric field within the grain-core. Moreover, the distance a photoelectron has to travel within the grain-to reach the surface-decreases. These effects have been modeled accurately by Watson (1972); however, the approximate model as suggested by Draine (1978) has been implemented into our numerical code:

$$
Y\left(r_{\text {grain }}\right)=\frac{\beta^{2}}{\alpha} \frac{\alpha^{2}-2 \alpha+2-2 \exp [-\alpha]}{\beta^{2}-2 \beta+2-2 \exp [-\beta]},
$$

where $\beta=r_{\text {grain }} / l_{\text {photon }}$ is the inverse penetration depth of the photon, and $\alpha=r_{\text {grain }} / l_{\text {photon }}+r_{\text {grain }} / l_{\text {electron }}$ relates the

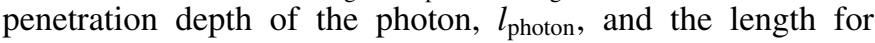
a photoelectron to travel within the grain-core, $l_{\text {electron}}$. This distance is assumed to be $l_{\text {electron }}=10 \AA$ and represents the distance from the molecular orbitals containing loosely bound electrons to the surface of the grain. The photon penetration depth is given by $l_{\text {photon }}=\lambda[4 \pi \Im(\tilde{n})]^{-1}$, where $\lambda$ is the wavelength of the impinging photon and $\Im(\tilde{n})$ is the imaginary part of the refractive index of the grain material. The total yield is then given by

$$
Y_{\mathrm{tot}}=Y(h v) Y\left(r_{\text {grain }}\right) .
$$

Photoelectrons with a total yield exceeding unity are always released. When the yield is less than unity a Monte Carlo approach is applied and a photoelectron only gets released if the determined yield exceeds a random number drawn from a normalized even distribution. 


\subsection{Initial Kinetic Energy of the Released Photoelectrons}

The distribution function for the initial kinetic energy of photoelectrons is assumed to be parabolically shaped as suggested by Weingartner \& Draine (2001) and a normalized distribution might be given by

$$
f(E)=\frac{-6 E^{2}+6 E E_{\text {high }}^{*}}{E_{\text {high }}^{* 3}},
$$

where $E_{\text {high }}^{*}$ is the excess energy of the impinging photon when the ionization energy has been subtracted, i.e., the highest possible kinetic energy a released photoelectron might get.

As the distribution is normalized for the interval $\left[0, E_{\text {high }}^{*}\right]$, a random number, $\mu$, between zero and one is used to set the kinetic energy, $E_{\mathrm{kin}}$, for the released photoelectron through the expression:

$$
\begin{gathered}
\mu=\int_{0}^{E_{\text {kin }}} \frac{-6 E^{2}+6 E E_{\text {high }}^{*}}{E_{\text {high }}^{* 3}} d E \\
E_{\text {kin }}=\operatorname{Root}\left(-6 E^{3} /\left(3 E_{\text {high }}^{* 3}\right)+6 E^{2} /\left(2 E_{\text {high }}^{* 2}\right)^{1 / 2}-\mu\right),
\end{gathered}
$$

where $\operatorname{Root}()$ is the root of the polynomial within the above stated energy interval.

\subsection{Dynamics of Electrons in the Electric Field of a Charged Dust Grain}

In the event that a photoelectron is released, a stationary positive charge is assigned to the center of the dust grain, which is assumed to be a spherical core with radius $R$. This radius is furthermore used to determine if an orbiting electron recombines with the charged grain-core, which is the case if it gets within a distance of $R$ from the center of the grain.

The electron emission site from the grain surface is selected randomly on the hemisphere facing the source. The site is obtained from a three component vector drawn from a Gaussian distribution scaled by the core radius. Under the assumption that the photon source is placed at $x=\infty$, it is only the half-sphere where $x$ is positive that undergoes emission processes and the site vector becomes $\left[\operatorname{Abs}\left(\hat{r_{x}}\right), \hat{r_{y}}, \hat{r_{z}}\right]$. Besides defining the emission site, the vector also represents the surface normal-the tangential plane at the emission site-used to determine the half-sphere in which the direction of the initial velocity vector $\left[\hat{v}_{x}, \hat{v_{y}}, \hat{v}_{z}\right]$ is restricted to.

Photoelectrons are assumed to escape from the charged dust grain electric field, provided they reach the boundary of the simulation cell. At this point, the background electric field is assumed to become dominant over the local field. To simulate the trajectory of the released electrons a Velocity Verlet algorithm (see the Appendix) and an accurate Coulomb potential are applied; the time step is $2.5 \times 10^{-15} \mathrm{~s}$. The grain-core is kept fixed in the center of the simulation cell.

\section{DUST GRAINS INTERACTION WITH YSOs' RADIATION FIELDS}

Four irradiation fields have been selected for this study. Two of them are blackbodies at temperatures of $50,000 \mathrm{~K}$ and $10,000 \mathrm{~K}$ simulating the photospheric radiation fields from $\mathrm{O}$ and $\mathrm{A}$ stars, respectively. A third spectrum is the fit to the interstellar radiation field made by Draine (1978) based

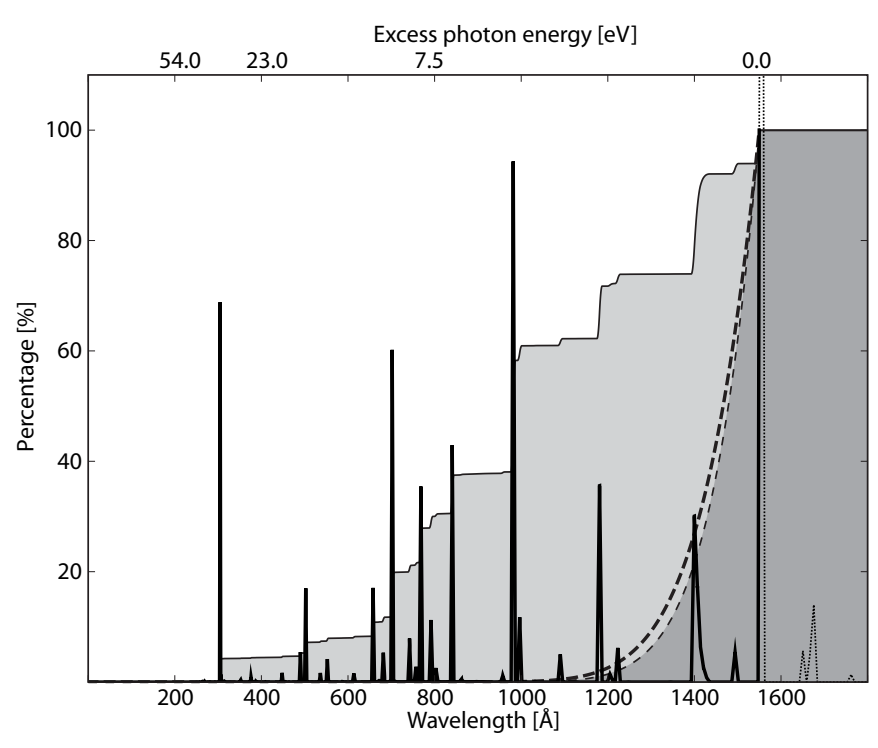

Figure 2. Distribution functions of the photon counts and the cumulative value for ionizing radiation. The solid line is the spectrum for a $\mathrm{T}$ Tauri star and the dashed line is the BB-50kK; both have been normalized to enclose a unit area. Gray-shaded areas are the cumulative percentage of the spectra below the specific wavelength.

on the early measurements of the Copernicus and Voyager missions (Habing 1968). This soft spectrum is used as a standard in chemical modeling of the disk; often the intensity of the radiation field is scaled with respect to the mean interstellar radiation field by means of the standard $G$ factor (typically $G=10^{4}$ at 100 AU, e.g., Semenov et al. 2004). Finally, a hard UV spectrum has been built for this study to model the hard energy radiative output of the TTSs. Note that there is little information on the spectral energy distribution of the TTSs in the range $10 \AA \leqslant \lambda \leqslant 950 \AA$. Furthermore, only few TTSs (TW Hya, RU Lup, and T Tau) have been observed in the 950-1100 $\AA$ range with the Far Ultraviolet Spectroscopic Explorer (Wilkinson et al. 2002; Herczeg et al. 2002). There are, however, strong evidences of energetic processes going on in the atmospheric/magnetospheric environment of the TTSs. On the one hand, the near UV $(1150 \AA \leqslant \lambda \leqslant 3200 \AA)$ indicates atmospheric structures extending to the inner border of the gas disk (see Gómez de Castro 2009 for a review). On the other hand, the observed X-ray luminosity of YSOs is on average about $10^{30} \mathrm{erg} \mathrm{s}^{-1}$ and the energy distribution is well fitted by the thermal radiation of optically thin plasmas at temperatures of 5-30 MK (see, e.g., Guedel 2008); for comparison the solar $\mathrm{X}$-ray luminosity is about $10^{27} \mathrm{erg} \mathrm{s}^{-1}$ and the plasma temperatures 1-2 MK. Thus, the propagation of the X-ray radiation on the dense $\left(\geqslant 10^{9} \mathrm{~cm}^{-3}\right)$ and extended $\left(2-4 R_{*}\right)$ atmospheres/ magnetospheres of the TTSs is expected to produce a rich and energetic spectrum. The spectrum used in this work has been used to fit the observed $\mathrm{C}$ IV/Si III] and $\mathrm{C}$ III]/Si III] lines ratios (Gómez de Castro \& Verdugo 2003).

The spectral energy distributions (in photons $\mathrm{s}^{-1} \mathrm{~cm}^{-2}$ ) have been normalized to the same bolometric flux. The ionizing part of the TTS model spectrum and the blackbody at 50,000 K are plotted in Figure 2; also, the cumulative distributions of ionizing photons are plotted for reference. Note that all the energy distributions are smooth besides the TTS distribution.

The simulated charging profiles of a $30 \mathrm{~nm}$ dust grain are shown in Figure 3 for the four energy distributions: blackbody at $50,000 \mathrm{~K}(\mathrm{BB}-50 \mathrm{kK})$, blackbody at $10,000 \mathrm{~K}(\mathrm{BB}-10 \mathrm{kK}), \mathrm{UV}$ 


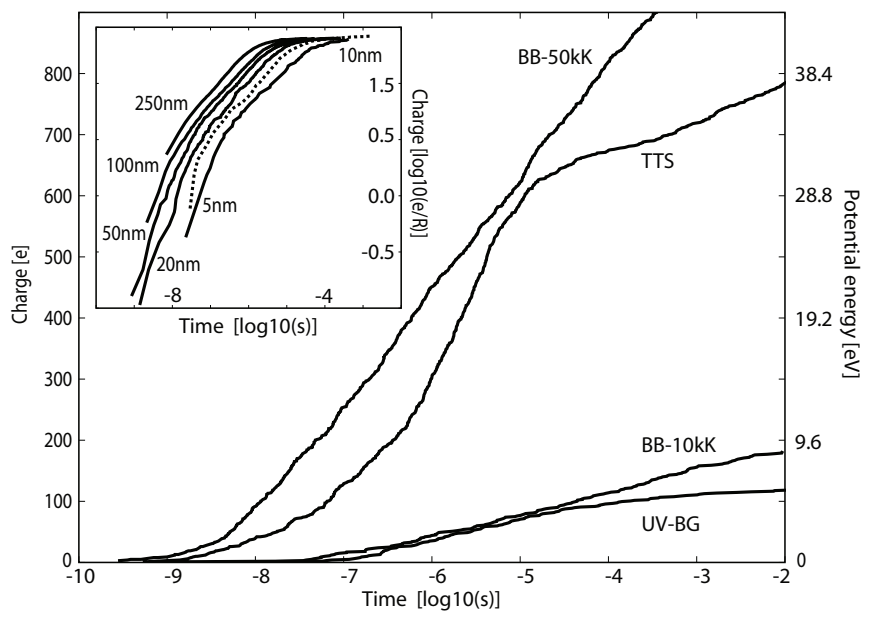

Figure 3. Charging profiles for a $30 \mathrm{~nm}$ grain when exposed to a spectrum from a T Tauri star (TTS), a blackbody spectrum at a temperature of either $10,000 \mathrm{~K}$ (BB-10kK) or $50,000 \mathrm{~K}$ (BB-50kK), and the UV background (UV-BG). It should be noted that for the BB spectra linear profiles are obtained whereas the TTS results in an S-shaped profile and the UV-BG saturates at a plateau level. Inset: charging profiles normalized with the grain radius for different grain sizes when exposed to the TTS spectrum.

background (UV-BG), and TTS. From the simulated results, it appears that the TTS spectrum gives rise to an S-shaped profile whereas linear profiles are obtained for the two blackbody spectra. The UV-BG saturates at a charge of approximately $125 e$. It also appears that the quantitative charging, when exposed to the TTS or the BB-50kK, is alike and that the UV-BG or $\mathrm{BB}-10 \mathrm{kK}$ gives rise to the similar charging rates. The relative slow charging obtained with the UV-BG or BB-10kK is a consequence of a low ratio of photons sufficiently energetic both to ionize an atom and to provide the needed kinetic energy for this released electron to escape the charged graincore, whereas the relative fast charging observed for the TTS or $\mathrm{BB}-50 \mathrm{kK}$ is possible because of a larger ratio of energetic photons.

The saturation threshold apparent in the figure for the UVBG spectrum is caused by the high energy cutoff of the energy distribution. The shortest wavelength corresponds to a photon energy of $14 \mathrm{eV}$, which gives an excess energy of $6 \mathrm{eV}$, equaling the needed kinetic energy an electron requires to escape the electric field created by a grain of charge $125 e$ and a radius of $30 \mathrm{~nm}$ that explains the observed plateau level. The linear profiles for the BB spectra are consistent with the experimental observation by Sickafoose et al. (2000) and is a behavior that is accounted for by theoretical models for the charging of a capacitor. From the linearity of the charging profiles it can be concluded that effects caused by the interactions between released electrons are negligible for the simulated photon fluxes. The S-shaped charging profile caused by TTS is a consequence of the line structure of this spectrum. From the cumulative distributions it also appears that the ratio of photons at high energies is larger in the TTS spectrum than in BB-50kK; see Figure 2. A consequence of this appears in the interval from $[1 \mu \mathrm{s}, 10 \mu \mathrm{s}]$ in Figure 3 where the TTS almost manages to reach the same charge state as obtained by the $\mathrm{BB}-50 \mathrm{kK}$. If the energy scale shown rightmost in Figure 3 is compared with the one uppermost in Figure 2, it emerges that the energy level at which the curvature changes after $10^{-5} \mathrm{~s}$ coincides with the first significant peak in the TTS spectrum.

\subsection{Dependence on the Size of the Dust Grain}

A series of simulations has been carried out to address the dependency between the grain size and the charging profile. Only the TTS spectrum is used for this purpose. As expected, the maximum charge a grain may achieve increases as the grain size increases. However, the maximal charge is determined by the radius of the grain rather than its volume; see inset in Figure 3. As a result, the maximal charge-to-mass ratio increases as the grains becomes smaller. This behavior is caused by the electric potential at the grain surface since the energy barrier an electron must overcome to escape the grain surface is $\propto Q / r_{\text {grain }}$.

\section{MODEL OF THE PHOTOELECTRIC EMISSION IN THE TTSs' ENVIRONMENT}

Two main results can be derived from the simulations: (1) the charging profile qualitatively remains unaffected when the grain size varies and (2) the maximal charge obtainable by a grain is solely determined by its radius. Thus, to reproduce the charging profile of an arbitrarily sized grain it is sufficient (1) to fit the general functional form of the charging profile for a grain and (2) to multiply it by a scaling factor, which is a linear function of the radius.

The charging profile of a fiducial $10 \mathrm{~nm}$ dust grain has been fitted. The grain is irradiated with photons from the TTS spectrum until it reaches a saturation threshold of $275 \mathrm{e}$. During the simulation, one to two electrons usually orbit the grain before they either escape or recombine with the grain core. Electrons leaving the computational box are lost to the background. The charging profile has been normalized to a photon rate, $S_{19}$, of $10^{19} \mathrm{~s}^{-1} \mathrm{~cm}^{-2}$ with energies above the dust grain threshold for photoionization that corresponds to photons with wavelengths $\lambda<1550 \AA$. This photon flux is equivalent to what is expected from an extended magnetosphere of radius $3 R_{\odot}$ producing a total ionizing luminosity of $0.03 L_{\odot}$. Note that, for the TTS spectrum, this photon rate is 4.8 times higher than the photon rate in the Lyman continuum $(1100 \AA<\lambda<912 \AA)$. The charging profile is represented in Figure 4 and the polynomial fit to it is given by

$$
\begin{aligned}
\log _{10} Q(x)= & -0.0015 x^{4}-0.00455 x^{3}-0.0177 x^{2} \\
& +0.0213 x+2.4326
\end{aligned}
$$

$x=\log _{10}\left(S_{19} t\right)=\log _{10}\left(\frac{S}{10^{19} \mathrm{~s}^{-1} \mathrm{~cm}^{-2}} \frac{t}{1 \mu \mathrm{s}}\right) \in[-8.4,-1.3]$.

The functional dependence for $Q\left(x, r_{\text {grain }}\right)$ is then described by

$$
Q\left(x, r_{\text {grain }}\right)=\frac{r_{\text {grain }}}{10 \mathrm{~nm}} \begin{cases}0 & \text { if } S_{19} t<4 \times 10^{-9} \\ Q(x) & \text { if } 4 \times 10^{-9} \leqslant S_{19} t \leqslant 5 \times 10^{-2} \\ 275 & \text { if } S_{19} t>5 \times 10^{-2} .\end{cases}
$$

For this grain size the asymptotic value for the final charge is set to $275 e$ as only photons with wavelengths less than $\sim 258 \AA$ will be able to cause any further charging that can be assumed to be of an insignificant rate; see Figure 2. The charging profile for the proposed analytical expression and the simulated data are shown in Figure 4. From the figure it appears that the proposed model reproduces the main features of the numerical 


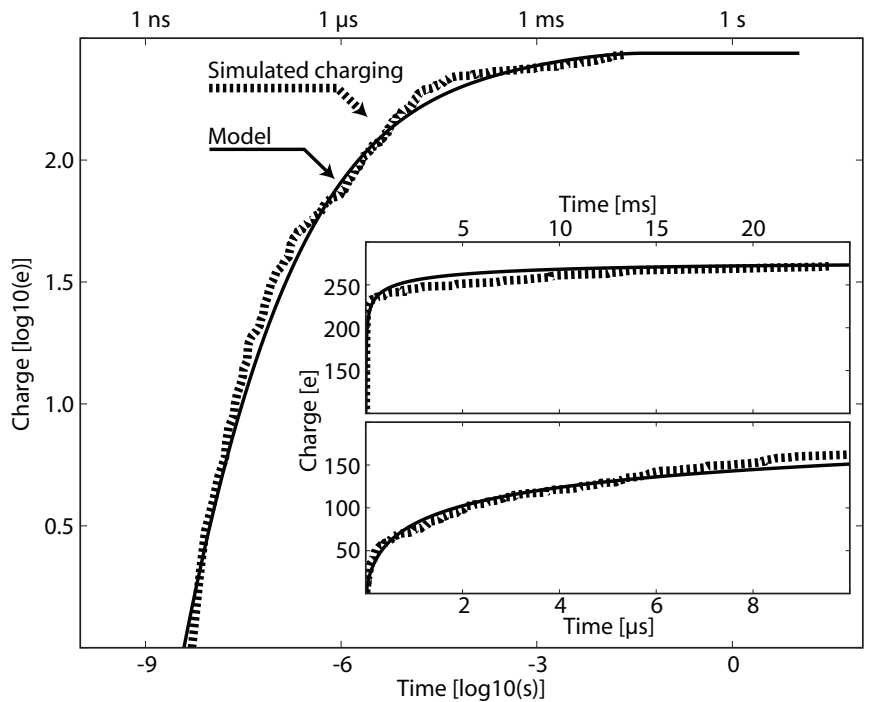

Figure 4. Charging model for an arbitrarily sized grain. The fourth-order polynomial used to model the dependence between time and the charge state of a grain are shown in a double logarithmic plot together with simulated data; the parameters used are as stated in Equation (11). It should be noted that the asymptotic plateau value used in the model is set to $275 e$. Insets show the simulated data and the fitted model for the charge state as a function of time.

simulations: an abrupt initial charging and a final plateau level. For the remaining intermediate interval a good agreement is obtained between the simple analytical model and the more accurate numerical results.

\subsection{The Dependence of the Photoelectric Current on the Grain Charge}

To derive a simple parameterization of $J_{e}^{o}$ on the grain charge $Q$, a new series of simulations was conducted where the grain cores are kept at a constant charge level. In these simulations, the kinetic energy of electrons escaping the grain is recorded; however, rather than losing these electrons to the background, they are reassigned to the grain-core to maintain its charge state. To generate the data, grains at different charge levels are exposed to the TTS spectrum for $1 \mu \mathrm{s}$. The exact same series of photons is used for each run. The number of ejected photoelectrons is found to decrease exponentially as $\propto 10^{-0.016 Q}$, where $Q$ is the charge of the dust grain and the current of photoelectrons, $J_{e}^{e}$, emitted from the $10 \mathrm{~nm}$ grain, might be expressed as

$$
J_{e}^{e}=6.3 \times 10^{2} \frac{\text { electrons }}{\mu \mathrm{s}} S_{19} 10^{-0.016 Q}
$$

Under the assumption that photoelectrons are emitted isotropically from the $10 \mathrm{~nm}$ dust grain surface, the photoelectric flux is given by

$$
\Phi_{e}^{e}=2 \times 10^{20} \frac{\text { electrons }}{\mathrm{cm}^{2} \mathrm{~s}} S_{19} 10^{-0.016 Q},
$$

which can be scaled as

$$
\Phi_{e}^{e}=2 \times 10^{20} \frac{\text { electrons }}{\mathrm{cm}^{2} \mathrm{~s}} S_{19}\left(\frac{r_{\text {grain }}}{10 \mathrm{~nm}}\right)^{-1} 10^{-0.016 Q}
$$

for any arbitrary radius of dust grains, where the dependence of the dust grain charge on the radius is inserted from Equation (13).

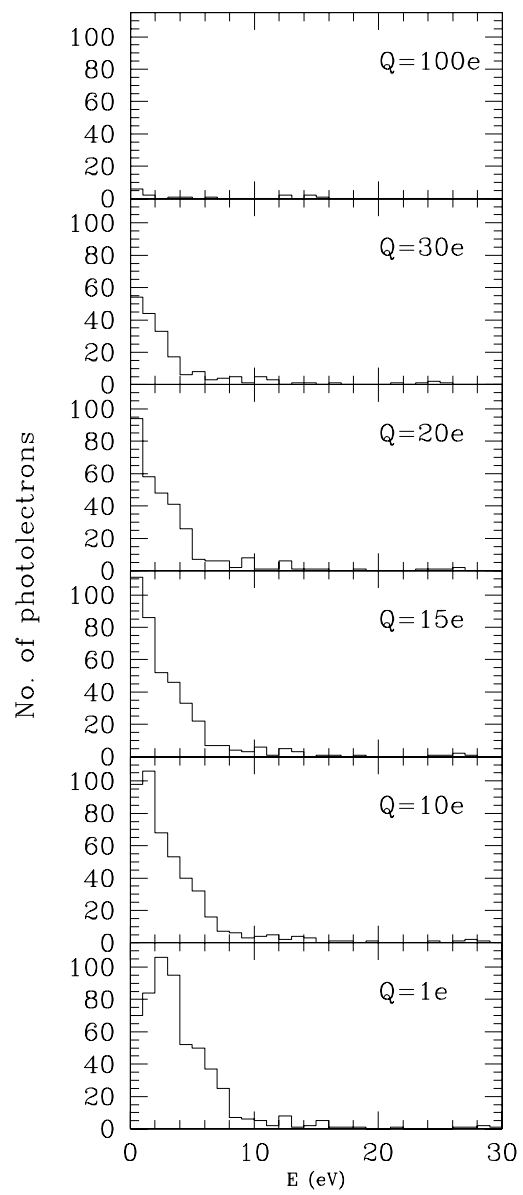

Figure 5. Energy distribution of the ejected photoelectrons. As the charge of the grain increases, only the high energy electrons are able to reach the boundary of the system and escape the grain-core.

\subsection{Energy Distribution of the Photoelectrons}

The energy distribution of the ejected photoelectrons follows a bimodal distribution with a narrow peak at about $2.5 \mathrm{eV}$ and a broad shoulder peaking at $\sim 5.5-6 \mathrm{eV}$. As the charge of the grain grows - which results in an increased local electric field-only electrons in the high energy tail of the distribution are able to escape and the electron flux decreases dramatically, as shown in Figure 5. This distribution is caused by the energy distribution of the TTS spectrum irradiating the dust grain; the first peak at $2.5 \mathrm{eV}$ is produced by the $\operatorname{Ly} \alpha$ continuum while the shoulder at $5.8 \mathrm{eV}$ is caused by high energy far-UV photons produced by highly ionized species in the $700 \AA-850 \AA$ wavelength range. $A$ useful parameterization of this behavior is given by

$$
\begin{aligned}
\frac{d J_{e}^{e}(Q, E)}{d E}= & 105 \exp \left(\frac{-\left(E^{\prime}-2.5\right)^{2}}{3}\right) \\
& +40 \exp \left(\frac{-\left(E^{\prime}-5.8\right)^{2}}{4}\right),
\end{aligned}
$$

where $E^{\prime}=E+0.14(Q-1)$ and $J_{e}^{e}(Q, E)$ is the number of photoelectrons ejected with energy in the range $(E-0.5$, $E+0.5) \mathrm{eV}$, for a grain with charge $Q$ and radius $10 \mathrm{~nm}$. The fraction of photoelectrons ejected with energies susceptible to ionize the hydrogen $(13.6 \mathrm{eV})$ is $3 \%$ for $Q=1$. The parameterization of the photoelectron energy distribution allows us to address the effect of the UV irradiation, in terms of the two electron populations produced: 
1. a low energy population with $E=2.5 \mathrm{eV}$ and dispersion $1.2 \mathrm{eV}$, and

2. a high energy population with $E=5.8 \mathrm{eV}$ and dispersion $1.4 \mathrm{eV}$,

where the dispersion is a measure of the temperature of the electron beam: $1.4 \times 10^{4} \mathrm{~K}$ and $1.6 \times 10^{4} \mathrm{~K}$ for the low and high energy populations, respectively. Note that the high energy population is well above the dissociation threshold of the $\mathrm{H}_{2}$ molecule $(4.52 \mathrm{eV})$ and may contribute to the ionization of $\mathrm{Mg}\left(\chi_{i}=7.64 \mathrm{eV}\right)$, which is a fundamental charge carrier for protostellar disks (Oppenheimer \& Dalgarno 1974; Ilgner \& Nelson 2008).

\section{DISCUSSION: DUST CHARGING IN TTSs’ DISKS}

The dust charging profile derived in Section 4 provides a useful parameterization of the photoelectric current generated by dust grains irradiated by a TTS spectrum. To determine the final charge of dust grains is a much more complex problem since other processes must be taken into account, including collisions with charged particles or charge transfer reactions between the dust grains and the surrounding molecules (see van Zadelhoff et al. 2003 and Semenov et al. 2004 for a detailed evaluation of the ionization degree and the main chemical processes in protostellar disks). For a generic electron density, $n_{e}$, and temperature, $T_{e}$, the final dust grain equilibrium charge is determined by the balance of the ejected photoelectric emission flux, $\Phi_{e}^{e}$, and the charge driven into the positively charged grain by the collisions with the surrounding thermal electrons. The flux of the absorbed electrons per unit surface on the dust grain, $\Phi_{e}^{a}$, (Spitzer 1978) is given by

$$
\Phi_{e}^{a}=\xi_{e} n_{e}\left(\frac{8 k T}{\pi m_{e}}\right)^{1 / 2}\left(1+\frac{e^{2} Q}{r_{\text {grain }} k T}\right)
$$

where $\xi_{e}$ is the sticking probability of the electrons striking the grain and $\left(8 k T / \pi m_{e}\right)^{1 / 2}$ is the thermal velocity of the electrons. For a $10 \mathrm{~nm}$ radius dust grain,

$$
\frac{e^{2} Q}{r_{\text {grain }} k T}=1.67 Q\left(\frac{10^{3} \mathrm{~K}}{T}\right)
$$

and thus,

$$
\Phi_{e}^{a}=6.4 \times 10^{7} \frac{\text { electrons }}{\mathrm{cm}^{2} \mathrm{~s}} \xi_{e} n_{e}\left(\frac{T}{10^{3} \mathrm{~K}}\right)^{1 / 2}\left(1+1.67 Q\left(\frac{10^{3} \mathrm{~K}}{T}\right)\right) .
$$

Both, $\Phi_{e}^{a}$ and $\Phi_{e}^{e}$ depend on the environmental conditions. Let us make an estimate of the relevance of photoelectric charging in the atmospheres of TTS disks.

\subsection{Physical Properties of $\alpha$-disks}

Assuming a simple $\alpha$-disk model (Shakura \& Sunyaev 1976), the variation of the disk density and temperature is readily prescribed in terms of $R$, the distance to the disk axis, and $z$, the height above the disk midplane for a given accretion rate, $\dot{M}_{a}$, and mass of the central star, $M_{*}$. The density of the disk, $n$, is given as

$$
n(R, z)=n(R, 0) \exp (-z / H(R))
$$

with the density in the disk mid-plane being

$$
\begin{aligned}
n(R, 0)= & 9.02 \times 10^{13} \mathrm{~cm}^{-3}\left(\frac{\dot{M}_{a}}{10^{-8} M_{\odot} \mathrm{yr}^{-1}}\right)^{5 / 8} \\
& \times\left(\frac{M_{*}}{M_{\odot}}\right)^{5 / 8}\left(\frac{R}{0.1 \mathrm{AU}}\right)^{-15 / 8}
\end{aligned}
$$

for $R \gg R_{*}$, as in this case, and assuming that the main component of the disk mass is molecular hydrogen. The scale height of the disk, $H(R)$, is given by

$$
\begin{aligned}
H(R)= & 3.1 \times 10^{10} \mathrm{~cm}\left(\frac{\dot{M}}{10^{-8} M_{\odot} \mathrm{yr}^{-1}}\right)^{1 / 8} \\
& \times\left(\frac{M_{*}}{M_{\odot}}\right)^{-3 / 8}\left(\frac{R}{0.1 \mathrm{AU}}\right)^{9 / 8} .
\end{aligned}
$$

The disk temperature at a given radius, $R$, is derived from the $\alpha$-disk prescription to be

$$
\begin{aligned}
\frac{T}{10^{3} \mathrm{~K}}= & 0.48\left(\frac{\dot{M}_{a}}{10^{-8} M_{\odot} \mathrm{yr}^{-1}}\right)^{1 / 4} \\
& \times\left(\frac{M_{*}}{M_{\odot}}\right)^{1 / 4}\left(\frac{R}{0.1 \mathrm{AU}}\right)^{-3 / 4} .
\end{aligned}
$$

\subsection{UV Irradiation of $\alpha$-disks}

$S_{19}$ varies several orders of magnitude within the dust disk and depends on the distance to the star and the opacity of dust grains to the UV radiation. UV radiation penetrates through the disk atmosphere creating a photodissociation region, which has been extensively studied (see, e.g., Hollenbach \& Gorti 2009). It is expected that the ionization front driven by the UV radiation splits the atmosphere into two regions, an upper ionized layer where $\mathrm{HI}$ is fully dissociated and a transitional region where the ionization fraction of $\mathrm{H}$ I decreases to negligible values and the electron density is controlled by $\mathrm{Mg}_{\mathrm{I}}$ ionization. Farther inside the disk, only X-ray photons are able to penetrate and ionize the dust grains.

Accretion disks around TTSs do not reach the stellar surface; the stellar magnetic field disrupts the inner area of the disk. Typically accretion disks are considered to extend from some 3-4 stellar radii to some $500 \mathrm{AU}$ (see, e.g., Millan-Gabet et al. 2007); however, the inner most area of the disk is devoid of dust grains. The UV radiation from the star melts the dust grains to a radius of $\sim 0.1-0.2 \mathrm{AU}$. This radius roughly corresponds to the silicates' melting radius. ${ }^{5}$ At this inner radius, $S_{19}=0.073$ and decreases steadily to $S_{19}=2.9 \times 10^{-9}$ at $500 \mathrm{AU}$ in the outskirts of the disk.

The stellar flux is absorbed by the ionized recombining upper layer and by dust absorption. The transfer of the stellar UV flux, $F_{\mathrm{UV}}$, in this layer is determined by

$$
\frac{d F_{\mathrm{UV}}(R, z)}{d z}=-\alpha_{B} n(R, z)^{2}-n(R, z) \sigma_{\mathrm{UV}} F_{\mathrm{UV}}(R, z),
$$

where $\alpha_{B}$ is the case $\mathrm{B}$ hydrogen recombination coefficient that at $10^{4} \mathrm{~K}$ is $\alpha_{B} \simeq 2.53 \times 10^{-13} \mathrm{~cm}^{3} \mathrm{~s}^{-1}$ (Storey \& Hummer

\footnotetext{
5 At thermal equilibrium, a dust grain irradiated by a TTS with luminosity $L_{*}$ is heated to a temperature $T$, such that $\pi r_{\text {grain }}^{2} L_{*} / 4 \pi R^{2}=4 / \pi r_{\text {grain }}^{2} \sigma T^{4}$. For $T$ about $2 / 3$ of the silicates' melting temperature $(T=2005 \mathrm{~K})$, the value of $R$ derived is $0.1 \mathrm{AU}$, for a solar luminosity star.
} 

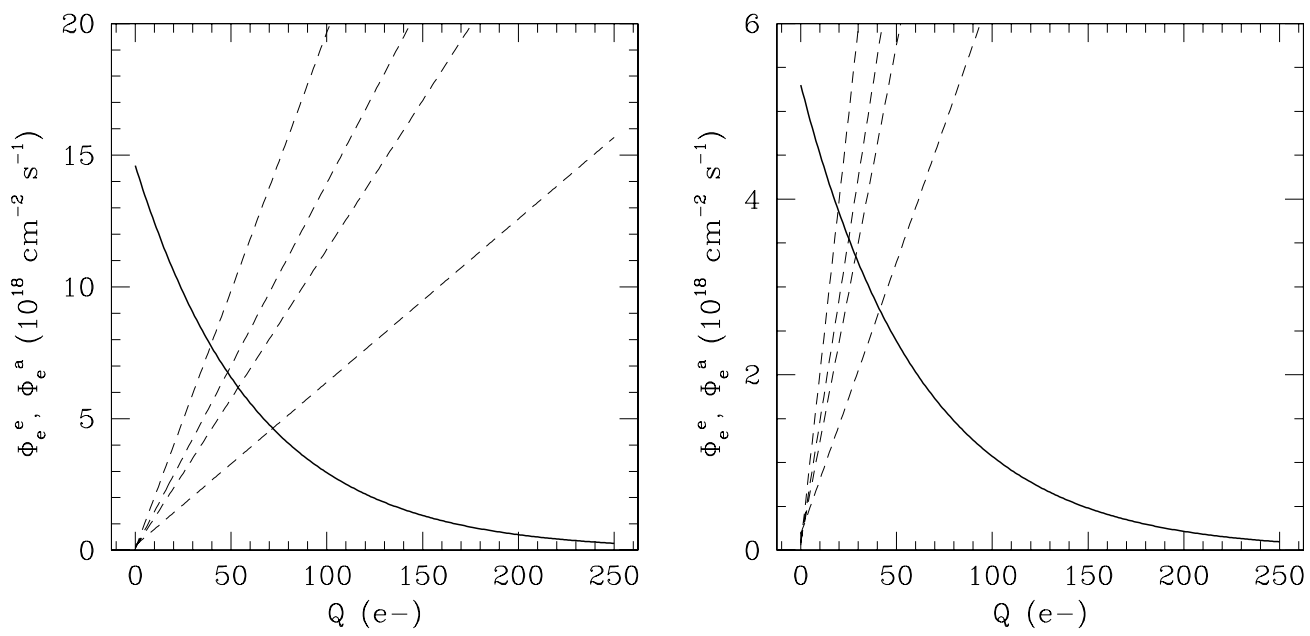

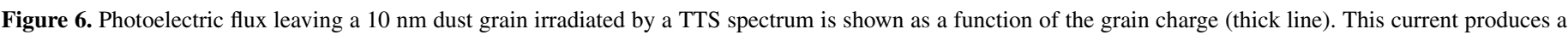

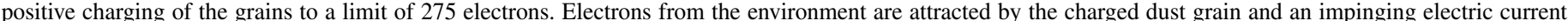

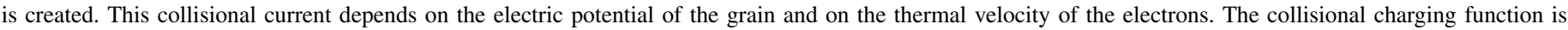

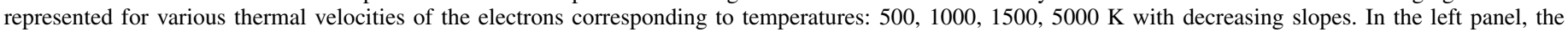
photoelectric flux, $\Phi_{e}^{e}$, is shown for $S_{19,0}=0.073$. In the right panel, the photoelectric flux, $\Phi_{e}^{e}$, is shown for $S_{19,0} / e=0.026$.

1995), $\sigma_{\mathrm{UV}}$ is the dust grains' absorption cross section per hydrogen nucleus, typically $\sigma_{\mathrm{UV}}=0.61 \times 10^{-21} \mathrm{~cm}^{-2}$ (Bertoldi $\&$ Draine 1996) and $n(R, z)$ is the hydrogen density at cylindrical coordinates $(R, z)$ in the disk. The general solution to this equation is

$$
F_{\mathrm{UV}}(R, z)=\exp ^{-\tau(z)}\left(F_{\mathrm{UV}, 0}-\int_{0}^{z} d z^{\prime} \alpha_{B} n^{2}\left(R, z^{\prime}\right) \exp ^{-\tau\left(z^{\prime}\right)}\right)
$$

with $\tau(z)=\sigma_{\mathrm{UV}} \int_{0}^{z} n\left(R, z^{\prime}\right) d z^{\prime}=\sigma_{\mathrm{UV}} N_{H}$ with $N_{H}$ the column density of $\mathrm{H}_{\mathrm{I}}$ and $F_{\mathrm{UV}, 0}$ the radiation field at the disk surface. However, the stellar radiation ought to cross the wind departing from the disk that may effectively shield the disk against photoionization at early stages (see Ferro-Fontán \& Gómez de Castro 2003 for an analysis of the effect caused by the fast centrifugally driven magnetic winds and Hollenbach \& Gorti 2009 for an analysis of the effect caused by slow, thermally driven winds). In such a case, the dominant absorbing component is $\mathrm{HI}$; an optical depth of 1 is reached for an $\mathrm{HI}$ column of $10^{17}-10^{18} \mathrm{~cm}^{-2}$. Moreover, the properties of dust grains in the disk atmospheres are ill determined but significant deviations from the standard dust grains size distribution in the interstellar medium are expected $;{ }^{6}$ large dust grains are expected to be more numerous due to the high densities in the disk.

In summary, in the absence of well-determined properties of the absorption of the UV flux on the disk atmosphere, $S_{19}$ can be parameterized as $S_{19,0} \exp ^{-\tau}$, where $S_{19,0}$ is the flux at the disk surface and $\exp ^{-\tau}$ parameterizes the extinction as a function of an unknown optical depth $\tau$. Note that the gas and dust columns are very high and that UV radiation will affect only the upper atmospheric layer. Typically, the dust grains' absorption cross section per hydrogen nucleus, $\sigma_{\mathrm{UV}}$, is $\sim 0.61 \times 10^{-21} \mathrm{~cm}^{-2}$ (Bertoldi \& Draine 1996). Thus, in the innermost region of an

\footnotetext{
6 Following Pollack et al. (1994), the dust particle size distribution in protostellar disks can be modeled as:

$N_{d}\left(r_{\text {grain }}\right)=N_{d, 0} r_{\text {grain }}^{-3.5}$, for $r_{\text {grain }} \in[0.005-1] \mu \mathrm{m}$ and

$N_{d}\left(r_{\text {grain }}\right)=N_{d, 0} r_{\text {grain }}^{-5.5}$, for $r_{\text {grain }} \in(1-5] \mu \mathrm{m}$.

According to this distribution, the average dust grain size is $\langle R\rangle=0.008 \mu \mathrm{m}$ and the maximum charge that such grains can achieve is $\left\langle Q_{\max }\right\rangle=220 e^{-}$.
}

$\alpha$-disk, the dust column reaches $\left(1 / \sigma_{\mathrm{UV}}\right)$ already at $z \sim 5 H$ (for a standard accretion rate of $10^{-8} M_{\odot} \mathrm{yr}^{-1}$ ).

\subsection{Relevance of Photoelectric Charging in the Atmosphere of an $\alpha$-disk}

Some hints on the relevance of photoelectric charging of dust grains in the accretion disks' atmospheres can be derived from a simple evaluation of $\Phi_{e}^{a}$ and $\Phi_{e}^{a}$ at $z=5 H$ for our fiducial dust grain of radius $10 \mathrm{~nm}$.

On the one hand, the collisional charging term, $\Phi_{e}^{a}$, can be derived using as fiducial density $n(R, 5 H)$ and deriving the thermal velocity of the gas from the temperature of the accretion disk $T(R)$ in the $\alpha$ prescription. Henceforth,

$$
\begin{aligned}
\Phi_{e}^{a}= & 8.3 \times 10^{18} \frac{\text { electrons }}{\mathrm{cm}^{2} \mathrm{~s}} \xi_{e} \chi_{e}\left(\frac{T}{10^{3} \mathrm{~K}}\right)^{1 / 2} \\
& \times\left(1+1.67 Q\left(\frac{10^{3} \mathrm{~K}}{T}\right)\right),
\end{aligned}
$$

where $\chi_{e}$ is the fraction of electrons in the atmosphere.

On the other hand, the photoelectric flux, $\Phi_{e}^{e}$, is given in terms of the stellar UV radiation flux entering the disk atmosphere, $S_{19,0}$ and the optical depth, $\tau$, as

$$
\Phi_{e}^{e}=2 \times 10^{20} \frac{\text { electrons }}{\mathrm{cm}^{2} \mathrm{~s}} S_{19,0} \exp ^{-\tau}\left(\frac{r_{\text {grain }}}{10 \mathrm{~nm}}\right)^{-1} 10^{-0.016 Q} .
$$

A simple illustration of the competition of both contributions, collisional charging versus photoelectric charge losses, is shown in Figure 6 for a $10 \mathrm{~nm}$ radius dust grains. In the top panel, $\Phi_{e}^{e}$ has been evaluated at the inner radius of the dusty disk, $0.1 \mathrm{AU}$, and plotted as a function of the grain charge. The rapid exponential decay is clearly seen. In the bottom panel, the stellar radiation has been assumed to be reduced by a factor of $1 / e$, as would correspond to $z=5 \mathrm{H}$. On top of that, the function of $\Phi_{e}^{a}(Q, T)$ has been plotted assuming in both cases that the ionization fraction is $\chi_{e}=0.01$, and that the sticking coefficient is $\xi_{e}=1$. $\Phi_{e}^{a}$ has been plotted for $T=500,1000$, 1200,1500 , and $5000 \mathrm{~K}$. The final charge of the dust grain is 
determined by the intersection between $\Phi_{e}^{e}$ and the $\Phi_{e}^{a}(Q, T)$ corresponding to the plasma temperature. As the temperature rises, the slope of $\Phi_{e}^{a}(Q, T)$ rises, and the equilibrium occurs at a larger charge for the same number of photoionizing photons, $S_{19}$. If $S_{19}$ decreases, the region of the exponential $\Phi_{e}^{e}$ curve sampled by the same family of $\Phi_{e}^{a}(Q, T)$ curves is at smaller values of $Q$.

Note that the temperature of the gas acts as a regulator of final dust grain charge and determines the region dominated by the photoelectric charging, as much as the photoionizing radiation flux.

\section{CONCLUSIONS}

The effect of the various UV radiation fields on dust grain charging has been analyzed by means of numerical simulations to show that TTS spectra are more effective in dust grain charging than the often applied UV-background. The photoelectric yield has two main populations, a low energy population of electrons with $E=2.5 \mathrm{eV}$ and a high energy population with $E=5.5-6 \mathrm{eV}$; the energy dispersion within the populations is $\sim 1.3 \mathrm{eV}\left(T \sim 1.5 \times 10^{4} \mathrm{~K}\right)$. The high energy population is susceptible to dissociating the $\mathrm{H}_{2}$ and ionizing some low ionization potential species, such as the $\mathrm{Mg}$. These results add an additional role to dust on the chemistry of the layers just below the $\mathrm{H}_{2}$ photoionization front.

Finally, a simple expression has been derived for the photoelectric currents from dust grains in TTS environments that can be introduced to the modeling of protostellar disks and a simple example of the relevance of photoelectric charging is presented for a rather naive $\alpha$-disk model.

This research project was funded by an UCM-EEA Abel Extraordinary Chair Grant. A.I.G. also acknowledges the support from the Spanish Ministry of Science and Innovation through grant AYA2008-06423-C03-01.

\section{APPENDIX}

Velocity Verlet, to start the simulation the initial position, $\mathbf{r}\left(t_{0}\right)$, and velocity, $\mathbf{v}\left(t_{0}\right)$, are required. When progressing the system in time, the acceleration, $\mathbf{a}\left(t_{n}\right)$, must be determined to update the position, $\mathbf{r}\left(t_{n+1}\right)$, and velocity, $\mathbf{v}\left(t_{n+1}\right)$, accordingly:

$$
\begin{aligned}
& \mathbf{r}\left(t_{n+1}\right)=\mathbf{r}\left(t_{n}\right)+\mathbf{v}\left(t_{n}\right) \Delta t+\mathbf{a}\left(t_{n}\right) \Delta t^{2} \\
& \mathbf{v}\left(t_{n+1}\right)=\mathbf{v}\left(t_{n}\right)+\frac{\mathbf{a}\left(t_{n}\right)+\mathbf{a}\left(t_{n+1}\right)}{2} \Delta t
\end{aligned}
$$

(see the appendix in Swope et al. 1982 for more details).

\section{REFERENCES}

Alexander, R., Clarke, J., \& Pringle, J. 2006, MNRAS, 369, 216

Bertoldi, F., \& Draine, B. 1996, ApJ, 458, 222

Draine, B. 1978, ApJS, 36, 595

Draine, B. 2003, ApJ, 598, 1026

Draine, B., \& Bertoldi, F. 1996, ApJ, 468, 269

Ferro-Fontán, C., \& Gómez de Castro, A. I. 2003, MNRAS, 342, 427

Gómez de Castro, A. I. 2009, Ap\&SS, 320, 97

Gómez de Castro, A. I., \& Verdugo, E. 2003, ApJ, 597, 443

Guedel, M. 2008, Astron. Nachr., 329, 218

Habing, H. 1968, Bull. Astron. Inst. Neth., 19, 421

Herczeg, G., Linsky, J., Valenti, J., Johns-Krull, C., \& Wood, B. 2002, ApJ, 572, 310

Hollenbach, D., \& Gorti, U. 2009, ApJ, 703, 1203

Ilgner, M., \& Nelson, R. 2008, A\&A, 483, 815

Ingleby, L., Calvet, N., Bergin, E., et al. 2009, ApJ, 703, L137

Millan-Gabet, R. F. M., Akeson, R., Leinert, C., et al. 2007, Protostars and Planets V (Tucson, AZ: Univ. of Arizona Press)

Oppenheimer, M., \& Dalgarno, A. 1974, ApJ, 192, 29

Pollack, J., Hollenbach, D., Beckwith, S., et al. 1994, ApJ, 421, 615

Raymond, J., Blair, W., \& Long, K. 1997, ApJ, 489, 314

Sargent, B., Forrest, W. J., Tayrien, C., et al. 2009, ApJ, 690, 1193

Semenov, D., Wiebe, D., \& Henning, T. 2004, A\&A, 417, 93

Shakura, N., \& Sunyaev, R. 1976, MNRAS, 175, 613

Sickafoose, A., Colwell, J., Horányi, M., \& Robertson, S. 2000, Phys. Rev. Lett., 84,6034

Spitzer, L. 1978, Physical Processes in the Interstellar Medium (New York: Wiley \& Sons), 199

Storey, P., \& Hummer, D. 1995, MNRAS, 272, 41

Swope, W., Andersen, H., Berens, P., \& Wilson, K. 1982, J. Chem. Phys., 76, 637

van Zadelhoff, G., Aikawa, Y., Hogerheijde, M., \& van Dishoeck, E. 2003, A\&A, 397, 789

Watson, W. 1972, ApJ, 176, 103

Weingartner, J., \& Draine, B. 2001, ApJS, 134, 263

Weingartner, J., Draine, B., \& Barr, D. 2006, ApJ, 645, 1188

Wilkinson, E., Harper, G., Brown, A., \& Herczeg, G. 2002, AJ, 124, 1077 